tionsverarbeitung (S. 120) oder auch der Online-Informationen als „Beziehungskiller“ (S. 126) auseinandersetzen.

Auch zahlreiche andere Kapitel des Buchs befassen sich mit der sog. interpersonellen Kommunikation, verstanden als Kommunikation zwischen Patienten und Ärzten oder allgemein zwischen Laien und Gesundheitsexperten, aber auch zwischen Laien und Laien oder zwischen den verschiedensten Angehörigen des Gesundheitswesens. Konkret für das Arzt/Patient-Verhältnis wird die Entwicklung weg von paternalistischen hin zu partizipativen Modellen einer Arzt/Patient-Interaktion nachgezeichnet, zu Recht aber auch kritisch hinterfragt, inwieweit diese Modelle überhaupt der Realität entsprechen und welche auch nachteiligen Konsequenzen damit möglicherweise verbunden sind. Der selbstsichere Patient ist nicht unbedingt ein $\mathrm{Ge}-$ winn für die Arzt/Patient-Beziehung - zumindest dann nicht, wenn dies auf Ärzteseite zu dem Versuch führt, die Kontrolle wieder zurückzugewinnen; solcherlei Interaktionen ,laufen erfahrungsgemäß spiralförmig abwärts im Sinne abnehmender Kommunikation und abnehmender Befriedigung im Arzt-Patienten-Gespräch“ (S. 307f.).

Diese wenigen Beispiele machen bereits deutlich, wie groß die Schnittmenge zwischen Gesundheitskommunikation und Recht ist. Dabei beschränkt sich die Schnittmenge keineswegs auf die Arzt/Patient-Kommunikation. Geht es um massenmediale Kommunikation zum Thema Gesundheit, tritt das Heilmittelwerberecht auf den Plan, geht es um Gesundheitskommunikation per Computer und Internet (s. dazu Teil VI des Werkes), erstreckt sich der rechtliche Fragenkatalog insbesondere auch auf haftungs- und datenschutzrechtliche Aspekte.

Im Vorwort zu diesem Sammelband ist davon die Rede, dass sich die Gesundheitskommunikation für viele Disziplinen zu einer wichtigen Inspiration entwickelt hat. Daran, dass diese Feststellung auch für die Disziplin der Rechtswissenschaft gilt, hat das hier besprochene Werk einen maßgeblichen Anteil.

DOI: $10.1007 /$ s00350-014-3888-8

\section{Die deutsche Stammzelldebatte. Eine exemplarische Untersuchung bioethischer Normenkonflikte in der politischen Kommunikation der Gegenwart.}

Von Alexandra Schwarzkopf. (Schriften zur politischen Kommunikation, Bd. 15). Verlag V \& $R$ unipress, Göttingen 2014, 316 S., geb., €44,99

Durch den rasanten Fortschritt der Medizin in den vergangenen Jahren beanspruchen bioethische Fragestellungen in ihrer rechtlichen und ethischen Tragweite eine zunehmende Relevanz. Die Debatte beschränkt sich seit langem nicht mehr auf die einschlägigen medizinischen Fachkreise, sondern ist längst zu einem übergreifenden interdisziplinären gesellschaftlichen Diskurs ausgewachsen. In ihrer Monographie, die im Jahre 2013 an den philosophischen und geschichtswissenschaftlichen Fakultäten der Universitäten Frankfurt a. M. und Trient (Italien) als Dissertation angenommen wurde, beschäftigt sich Alexandra Schwarzkopf mit der deutschen Stammzelldebatte, einem der wohl bedeutsamsten Konflikte der Bioethik der vergangenen Jahrzehnte. Im Fokus ihrer Ausarbeitung steht die dem im Jahre 2002 erlassenen und 2008 novellierten Stammzellengesetz (StZG) vorausgegangene Diskussion. Schwarzkopf untersucht in fünf Kapiteln die verschiedenen Aspekte des pluralistischen Kommunikationsprozesses. Ziel ihrer Arbeit ist die historische Aufarbeitung und Analyse der Debatte in der Erwartung, dass der medizinische Fortschritt auch in naher Zukunft weitere konfliktträchtige Themen zu Tage fördert, für die in einem politischen Prozess Entscheidungen getroffen werden müssen.

Eingangs erfolgt eine Übersicht über die einschlägigen gesetzlichen Regelungsinhalte (Kap. 2). Die Verfasserin stellt die Neuerung des StZG detailliert vor und ordnet sie in den historischen Kontext ein. Als maßgebliche Vorreiter erachtet sie die Regelungen zum

Wiss. Mitarb. Charlotte Landwehr,

Köln, Deutschland
Schwangerschaftsabbruch und die Regelung des ESchG, welche als gemeinsamer Nenner eine grundrechtliche Kollision der mütterlichen Position einerseits und der des ungeborenen Lebens andererseits vereint. Hierbei treffen Grundrechte von elementarer Bedeutung wie die Menschenwürde, die Selbstbestimmung und das Recht auf Leben und körperliche Unversehrtheit aufeinander. Eine vertiefte Auseinandersetzung erfährt die zentrale Frage nach der Grundrechtsträgerschaft der Blastozyste, deren Bejahung zur Folge hat, dass sich die Stammzelldebatte ausschließlich um die Zulässigkeit des Imports embryonaler Stammzellen und niemals um deren Gewinnung im innerdeutschen Raum dreht. Die seit den achtziger Jahren kontrovers diskutierte Frage stellt Schwarzkopf anschaulich dar, ohne selbst Position zu beziehen.

Das Herzstück ihrer Ausarbeitung widmet die Verfasserin den verschiedenen Standpunkten der Gesetzgebungsdebatte (Kap. 3). Ausführlich beleuchtet sie nacheinander die Positionen der Mitglieder des Bundestages, der wissenschaftlichen Experten in den Fachausschüssen, der Kommissionen und Räte, der Zentralen Ethikkommission und der Deutschen Forschungsgemeinschaft, der Kirchen und schließlich der Medienvertreter. Hierbei untersucht sie akribisch die einzelnen Diskussionspunkte der Stammzelldebatte und verdeutlicht die verschiedenen Positionierungen u. a. anhand von Redebeiträgen, Stellungnahmen und eigenen Interviews. Anknüpfend an die Grundlagen des ersten Kapitels sind die zentralen Themen der Stammzelldebatte erneut vornehmlich verfassungsrechtlicher Natur; exemplarisch hervorzuheben sind der Beginn des menschlichen Lebens, die Sorge um die Frau als „Eizelllieferantin“ und der Vergleich des Schutzniveaus des Embryos in vitro und in vivo. Auch die Frage nach der „Ethik des Heilens“, mithin ob und inwiefern der ärztliche Heilauftrag die Tötung und Instrumentalisierung des ungeborenen Lebens legitimiert, wird zu einer zentralen Frage. Trotz bestehender Divergenzen zwischen den einzelnen Kommunikationsteilnehmern eint diese bei einer im Grundsatz eher liberalen Tendenz, wie auch im Rahmen des Schwangerschaftsabbruches und insbesondere der PID, die Sorge vor einem „Dammbruch“.

Diese Furcht findet eine nähere Betrachtung in Kapitel 4. Alexandra Schwarzkopf konstatiert keine polarisierende, emotional aufgeladene Debatte, sondern erkennt eine auffällige Behutsamkeit und Zurückhaltung der einzelnen Teilnehmer und eine allübergreifende Kompromissbereitschaft mit dem Bestreben, rasch eine nachhaltige gesetzliche Regelung zu schaffen. Neben der Hochrangigkeit der durch die Stammzellenforschung tangierten Rechtsgüter sieht sie hierfür die nationalsozialistische Unrechtsvergangenheit Deutschlands sowie die Positionierung der beiden großen Kirchen als maßgeblich an. Schließlich schreibt sie Deutschland eine besondere „Debattenkultur“ zu, was sich in dem Bedürfnis nach einer gesamtgesellschaftlichen Diskussion ausdrückt. Diesen Eindruck vertieft sie in dem letzten Teil ihrer Arbeit, der einen rechtsvergleichenden Teil zu Stammzelldebatten in anderen Ländern wie Großbritannien und den USA beinhaltet.

Der Verfasserin gelingt eine fundierte Darstellung und Auswertung des Kommunikationsprozesses zur Stammzelldebatte. In Anbetracht zahlreicher weiterer konfliktträchtiger Themen, wie beispielsweise der rechtlichen Bewertung sog. Biobanken, die in naher Zukunft einen ähnlichen interdisziplinären Austausch fordern, erweist sich der Wert dieser Monographie nicht nur für die Politikwissenschaft. Das Werk verdeutlicht, dass sich Kollisionen elementarer verfassungsrechtlicher Güter grundsätzlich einem allgemeinen Konsens entziehen und dass angesichts des Wertepluralismus der Kompromiss der einzig gangbare Weg ist. Jüngst hat dies sehr anschaulich das Gesetzgebungsverfahren zur rechtlichen Regelung der Präimplantationsdiagnostik (PID) demonstriert. Zu hoffen bleibt, dass die Teilnehmer politischer Willensbildungsprozesse dieses Verfahren als Anregung sehen, eine offene Kommunikation zu führen, und so ein zielführendes „Miteinander statt ein Gegeneinander“ der verschiedenen Interessenvertreter entsteht.

Die Dissertation verfolgt nicht das Ziel, Antworten auf aktuelle juristische Fragestellungen zu geben. Der Wert und Gewinn auch für Juristen liegt darin, dass die Hintergründe des Gesetzgebungsverfahrens zum StZG exemplarisch verdeutlicht werden. Es handelt sich um ein aufschlussreiches Buch, dessen Lektüre gerade auch im Hinblick auf die anstehenden bioethischen Normenkonflikte sehr empfehlenswert ist. 